\title{
Case report: Image in Electrophysiology
}

\section{Cryoablation of para-Hisian accessory pathway in a child without fluoroscopy}

Ayan Abdrakhmanov, Abay Bakytzhanuly, Zhandos Esilbayev, Azat Tursunbekov, Omurbek Nuralinov, Alihan Baidauletov, Serik Bagibayev

JCS "National Research Cardiac Surgery Centre", Astana, Kazakhstan

\section{Abstract}

The case describes a successful cryoablation of para-Hisian accessory pathway in an adolescent.

(Heart Vessels and Transplantation 2017; 1: doi: 10.24969/hvt.2017.34)

Keywords: Para-Hisian accessory pathway, child, Wolff-Parkinson-White syndrome, cryoablation

A 14-year-old boy with Wolff-Parkinson-White syndrome underwent an electrophysiology study (EPs) for symptoms of palpitations and persistence of preexcitation. He was detected to have right postero-septal or mid-septal accessory pathway on electrocardiogram ECG (Fig.1). Patient had no previous ablation and antiarrhythmic therapy with amiodarone and beta-blockers were not effective. In a view of symptomatic palpitations in association with evidence of preexcitation, he underwent an EPs (Fig.2). EPs performed under general anesthesia. 3D mapping of the heart with navigation system the Saint Jude Medical (SJM) Velocity identified an earliest accessory pathway (AP) potential in para-Hisian area (Fig. 3). AP effective refractory period was $235 \mathrm{~ms}$, shortest pre-excited R-R interval (SPERRI) $225 \mathrm{~ms}$, radiofrequency ablation was performed around para-Hisian area with low power ( $\mathrm{P}$ 20-25W, t0C-50C) in postero-septal and mid-septal areas with no effect. The AP was too close to His bundle and we decided to perform cryoablation using created 3D anatomical map. During the cryomapping application $\left(-30^{\circ}\right)$ there was an effect (Fig. 4), after total cryoablation the pre-excitation disappeared (Fig. 5). In this case we used Medtronic cryocathether FreezorTM 7 Fr. Distal electrodes of the cryocathether were connected to the pin pad of navigation system SJM Velocity which allowed us to visualize the only tip of the cryocathether. The procedure was performed during the sinus rhythm with parameters: $240 \mathrm{sec},-420 \mathrm{C}$. Cryoablation was successful with no complication. Adenosine test showed total atrioventricular (AV) block, which confirmed the effectiveness of the cryoablation (Fig. 6). The procedure lasted 97 minutes (from time of the puncture till the decannulation), no fluoroscopy was used. At 6-month follow-up, he continues to have no pre-excitation on ECG. Focal cryoablation of the paraHisian AP can be safely performed in children to avoid AV block.

Peer-review: Internal

Conflict of interest: None to declare Authorship: A.A., A.B., Z.E., A.T., O.N., A.B., S.B. equally contributed to management of case and preparation of report

Acknowledgement and funding: None to declare

Address for Correspondence: Ayan Abdrakhmanov, Department of Interventional Arrhythmology, National Research Cardiac Surgery Centre, Astana, Kazakhstan Email: ayan-3@mail.ru

Received 12.12.2017 Accepted: 12.12.2017

Copyright@2017 Heart Vessels and Transplantation 




Figure 1. Surface electrocardiogram: PQ- $100 \mathrm{~ms}$, heart rate $-85 \mathrm{bpm}$, speed - $25 \mathrm{~mm} / \mathrm{s}$.



Figure 2. Electrogram and ECG tracing before ablation: $150 \mathrm{~mm} / \mathrm{s}, \mathrm{PQ}-106 \mathrm{~ms}, \mathrm{AV}-76 \mathrm{~ms}$. AV - Atrioventricular, ECG- electrocardiogram 


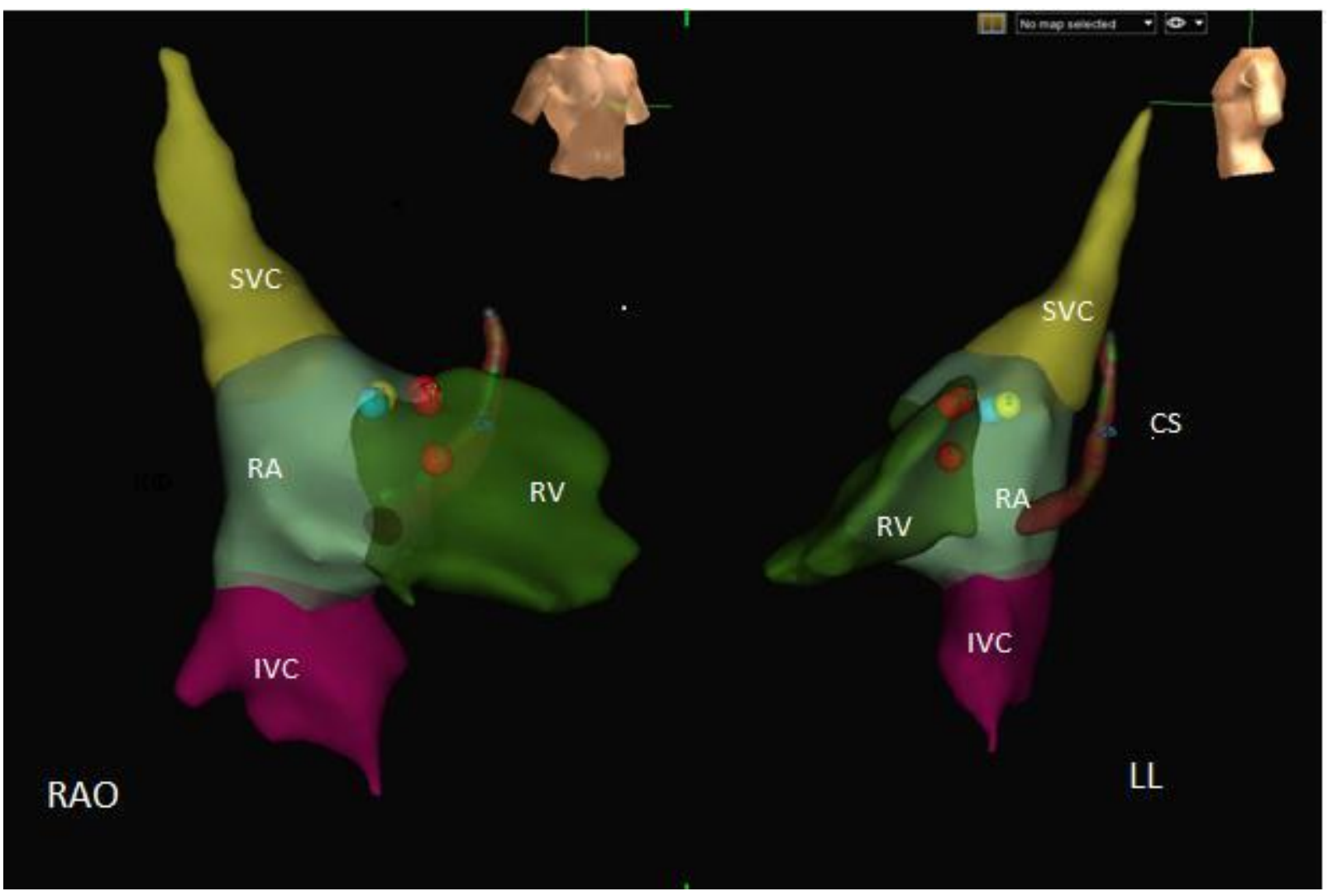

Figure 3. Focal cryoablation of para-Hisian AP. Navigation system SJM Velocity: yellow point - His, Red points - RF ablations, Blue point -cryoablation AP - accessory pathway, AV - atrioventricular, CS- coronary sinus, IVC - inferior vena cava, RA - right atrium, RV - right ventricle, RFradiofrequency, SVC - superior vena cava

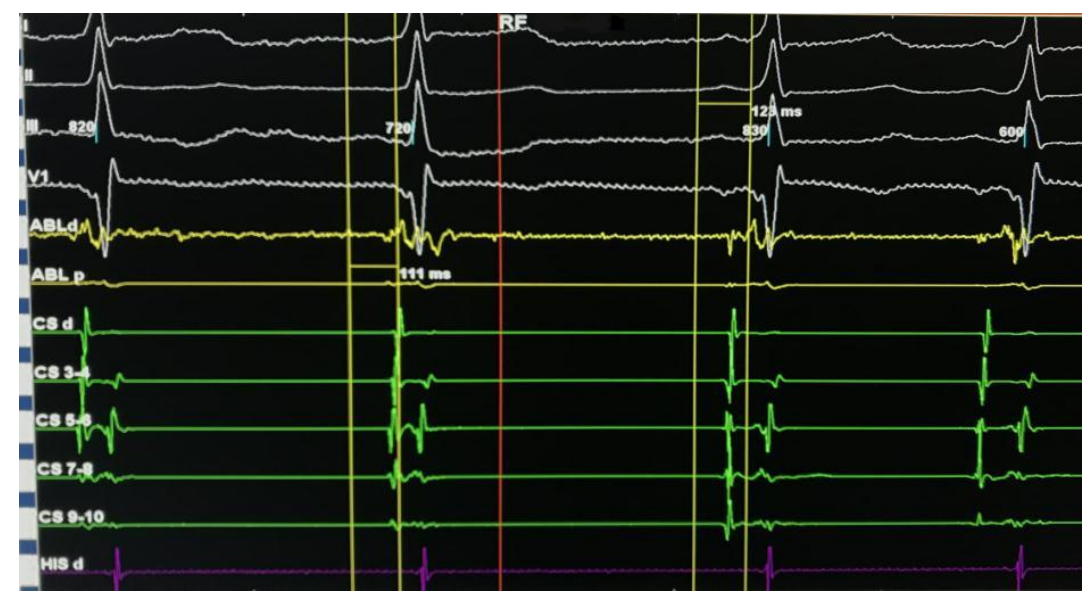

Figure 4. Focal cryoablation of para-Hisian AP - cryomapping (-30 $)$ application. PQ prolongation from $111 \mathrm{~ms}$ to $123 \mathrm{~ms}$, $150 \mathrm{~mm} / \mathrm{s}$.

AP - accessory pathway 


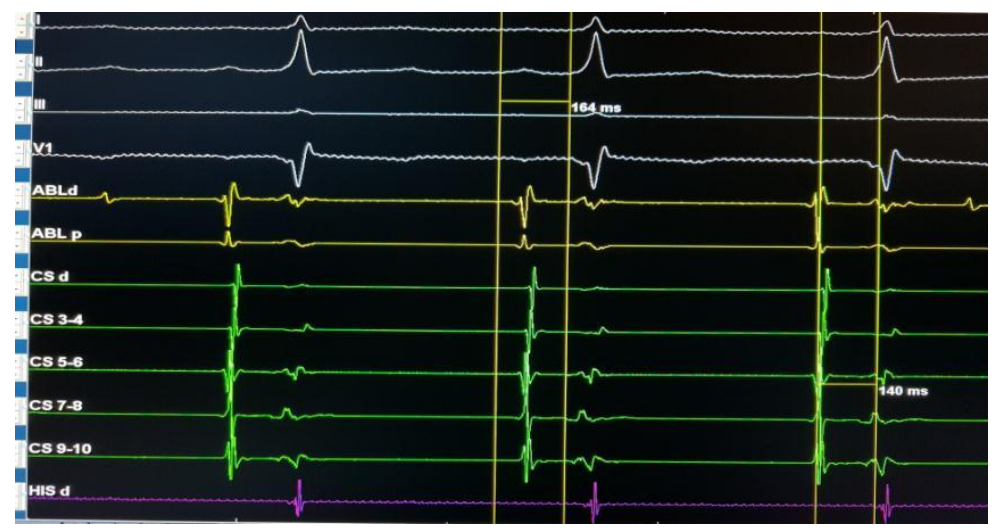

Figure 5. Focal cryoablation of para-Hisian AP. Effective cryoablation. PQ- $164 \mathrm{~ms}$, AV- $140 \mathrm{~ms}, 150 \mathrm{~mm} / \mathrm{s}$. AP - accessory pathway, AV - atrioventricular



Figure 6. Focal cryoablation of para-Hisian AP. Adenosine test. Drug induced total AV block, $150 \mathrm{~mm} / \mathrm{s}$. $\mathrm{AP}$ - accessory pathway, AV - atrioventricular 